reine Obligationen-Position gebracht werden. Die Familien- und die Geschäfts-

sphäre sind familienrechtlich getrennt und müssen es schuldrechtlich bleiben. Daran ändert auch die symbolische Beteiligung an Gesellschaftsvermögen nichts, die eingegangen wird, um den Formerfordernissen des Gesellschaftsrechts zu genügen. Dasselbe gilt für Kinder, die ihre eigene berufliche Existenz und ihre eigene Wohnung einrichten müssen: Es ist sittenwidrig im Sinne des $\$$ I 38 BGB, sie zu verpflichten, für die Geschäfts- und Lebenssphäre ihrer Eltern einzustehen, aus der sie sich emanzipieren müssen und zu der sie keinen existentiellen Bezug (mehr) haben. Es ist "für eine Rechtsgemeinschaft unerträglich «" ${ }^{34}$, wenn Banken als Stifter von Familienrechtsverhältnissen auftreten, die das Gesetz in den letzten Jahrzehnten zu ändern gezwungen war.

\title{
Christoph Schmidt-Eriksen Planfeststellungsverfahren Schacht Konrad: Die Crux mit der UVP
}

\section{Zum Hintergrund}

Am 24. Januar 199I erteilte Bundesumweltminister Töpfer der Niedersächsischen Umweltministerin Griefahn unter Berufung auf Art. 85 Abs. 3 GG die Weisung, in dem Planfeststellungsverfahren zur Genehmigung des atomaren Endlagers Schacht Konrad mit dem Verfahren der Öffentlichkeitsbeteiligung zu beginnen. Vorausgegangen war dem eine längere Auseinandersetzung zwischen dem Niedersächsischen Umweltministerium (NMU) und dem Bundesumweltministerium (BMU) darüber, ob die Planunterlagen auslegungsreif seien. Während sich das NMU darauf berief, $\mathrm{daß}$ von der Antragstellerin (die Bundesrepublik, vertreten durch das Bundesamt für Strahlenschutz (BfS), also eine dem BMU nachgeordnete Behörde) noch eine Umweltverträglichkeitsstudie nach $\$ 6 \mathrm{Abs} .3 \mathrm{Nr} .4$ UVPG vorgelegt werden müsse, hielt der BMU dies angesichts der bereits von der BfS vorgelegten Plan- und Genehmigungsunterlagen nicht für erforderlich. Stützen konnte er sich hierbei auf ein Schreiben des NMU vom is. Juni 1990, mit dem übereinstimmend der BMU dekretierte, er halte die Unterlagen für vollständig und auslegungsreif.' Pikanterweise datiert dieses Schreiben damit nur 6 Tage vor Konstituierung der neuen Rot-Grünen Landesregierung, nachdem das Verfahren bereits seit dem 3 I. Aug. 982 (Datum der Antragstellung auf Einleitung des Planfeststellungsverfahrens) anhängig war. ${ }^{2}$

Ziel der Umweltverträglichkeitsprüfung (UVP) eines technischen Großprojektes ist es, seine Umweltauswirkungen insgesamt zu erfassen. Die UVP dient der frühzeitigen und umfassenden Umweltvorsorge, damit das Ergebnis dieser Prüfung bei allen behördlichen Entscheidungen über die Zulässigkeit berücksichtigt werden kann (vgl. \$ I UVPG). Im Unterschied zu den materiellen Genehmigungsvorschriften spezieller Umweltgesetze, läßt sich aus dem UVPG ein ökosystemarer Ansatz ableiten ${ }^{3}$,

34 Mayer-Maly in: Münchener Kommentar zum Bürgerlichen Gesetzbuch, Band I, 2. Aufl. 1984, $\$_{13} 8$, Rdnr. I.

I Vgl. den Sachverhalt zum Urteil des BVerfG im Nieders. Weisungsstreit. BVerfGE 84, 25 (26)

2 Auch der vom BfS ausgelegte Plan läßt etwas von den Terminnóten nach der nieders. Landtagswahl 1990 erahnen. Er gibt an: $n$ Stand $9 / 86$ in der Fassung $4 / 90 \%$.

3 Vgl. hierzu Peters, Zum gesamthaften Prufungsansatz der EG-Richtlinie uber die Umweltverträglichkeitsprüfung. UPR I990, 133 , sowie Gassner, Die Medien- und Verfahrensübergreifende Umweltverträglichkeitsprüfung, UPR $1990,363$. 
der die natürliche Umwelt des Menschen in ihren verschiedentlichen Teilbereichen als vernetztes und zusammengehöriges Gesamtsystem versteht (vgl. $\$ 2$ Abs. I S. 2 UVPG). Das AtG etwa weist demgegenüber als Schutzgüter nur Leben, Gesundheit und Sachgüter $(\$$ I Nr. 2) aus. Belange des Natur- und Umweltschutzes finden zwar mittelbar Berücksichtigung in $\ 7$ Abs. 2 Nr. 6 AtG, wonach überwiegende öffentliche Interessen, insbesondere im Hinblick auf die Umweltauswirkungen, der Wahl des Standorts der Anlage nicht entgegenstehen dürfen. Unterdessen verdankt sich diese klarstellende Formulierung, die den rechtstechnischen Anknüpfungspunkt für die Integration der UVP in das atomrechtliche Genebmigungsverfahren bildet, erst der Einführung der UVP in das deutsche Genehmigungsrecht. ${ }^{4}$ Für $\$ 7$ Abs. 2 Nr. 6 AtG a.F. war es noch streitig, ob damit mehr als die nuklearspezifischen Risiken für die öffentlichen Interessen an der Reinhaltung des Wassers, der Luft und des Bodens im Hinblick auf den jeweiligen speziellen Standort der Anlage angesprochen waren.'

Die UVP findet unter Einbeziehung der Öffentlichkeit statt (\$9 UVPG). Aufgrund der Entscheidung des bundesdeutschen Gesetzgebers, sie als einen unselbständigen Teil verwaltungsbehördlicher Verfahren $(\$ 2$ Abs. I S. I) in bestehende Verfahren zu integrieren ${ }^{6}$, fallen die schon bislang bekannte Öffentlichkeitsbeteiligung in Planfeststellungs-7 und sonstigen Genehmigungsverfahren ${ }^{8}$ und die Öffentlichkeitsbeteiligung nach dem UVPG zusammen. Die Erörterung dient also beidem, sowohl der Klärung, inwieweit die Belange Dritter durch die Genehmigung der Anlage berührt sein können, als auch der Auseinandersetzung mit Dritten über die Umweltauswirkungen des Vorhabens. ${ }^{9}$

\section{Genehmigungstatbestand und Genehmigungsumfang für das Endlager Schacht Konrad}

Grundlegende Genehmigungsnorm für ein atomares Endlager ist $₫ 9 \mathrm{~b}$ AtG. ${ }^{1 \circ} \mathrm{Die}$ Errichtung und der Betrieb eines Endlagers für atomare Abfälle, von $\$ 9$ a Abs. 3 AtG zur Aufgabe des Bundes gemacht, bedürfen der Planfeststellung, $₫ 9 \mathrm{~b}$ Abs. I, bei der, wie Abs. 2 klarstellt, die Umweltverträglichkeit der Anlage zu prüfen ist. Die Umweltverträglichkeitsprüfung ist damit Voraussetzung für die Erteilung des Planfeststellungsbeschlusses (vgl. $\$ 9$ b Abs. 2 S. 2 i.V.m. $\$ 9$ b Abs. 4 AtG).

Ob die materiellen Genehmigungsvoraussetzungen eines Gesetzes erfüllt sind, kann nur in einem Verwaltungsverfahren geklärt werden. Das atomrechtliche Planfeststellungsverfahren richtet sich grundsätzlich nach den $\$ \$ 72 \mathrm{ff}$ des VwVfG; Abweichun-

4 Artikel 3 Nr. I des Gesetzes zur Umsetzung der Richtlinie des Rates vom 27. Juni 1985 über die Umweltverträglichkeitsprüfung bei bestimmten offentlichen und privaten Projekten $(8 \mathrm{~s} / 337 / \mathrm{EWG})$ vom 1 2. Februar 1990, BGBl., S. 20 s.

s Vgl. Haedrich, AtG, $\$ 7$ Anm. 19 m.w. N. zu Rspr. und Schrifttum.

6 Vgl. Jarass, Grundstrukturen des Gesetzes uber die Umweltverträglichkeitsprúfung, NuR 1991, 201 (202).

7 Vgl. hierzu nur die Untersuchungen zur "Öffentlichkeitsbeteiligung bei der Genehmigung von umweltrelevanten Großvorhaben «, Speyerer Forschungsberichte 70 , 1988.

$8 \mathrm{Z}$. B. im atomrechtlichen Genehmigungsverfahren fur Anlagen nach $₫ 7$ Abs. I und s gem. der AtVfVund im immissionsschutzrechtlichen Verfahren fur in der ${ }_{4}$. BImSchV festgelegte Anlagen gem. der 9. BImSchV.

9 Das gilt jedenfalls dann, wenn $\$ 9$ UVPG konform zur EG-Richtlinie zur UVP (Richtlinie des Rates uber die Umweltverträglichkeitsprüfung bei bestimmten öffentlichen und privaten Projekten vom 27. Juni $1985(8 \varsigma / 337 /$ EWG $)$, ABL. EG Nr. L 175 v. 5. 7.1985, S. 40 ) ausgelegt wird. Kritisch zu der mit dem Verweis des $\$ 9$ UVPG auf $\$ 73$ Abs. 3-7 VwVfG einhergehenden Beschrankung des Gegenstands und Umfangs der Erorterung Erbguth/Schink. UVPG, \$9 Anm. $8 \mathrm{ff}$ (Insbes. 10 ).

10 Die Entsorgungsregelungen der $\$ \$$ a ff sind mit der 4. Novelle zum AtG - Viertes Gesetz zur Änderung des Atomgesetzes vom 30. August 1976, BGBI. I, S. 2573 - in das Gesetz eingefugt worden. 
gen statuiert allerdings $₫ 9 \mathrm{~b}$ Abs. $\varsigma$ AtG: im Großen und Ganzen sollen die spezielleren verfahrensrechtlichen Regelungen der AtVfV den Verfahrensregeln der $\$ \$ 72 \mathrm{ff}$ $V_{w} V f G$ vorgehen $(\mathrm{Nr} . \mathrm{x})^{11}$ und Genehmigungen nach dem BBergG werden nicht mit der Entscheidung über den Plan festgestellt (Nr. 3).

\section{Konzentrationswirkung des Planfeststellungsverfabrens}

Das Planfeststellungsverfahren ist ein aus langer deutscher Verwaltungstradition entwickeltes Verwaltungsverfahren. ${ }^{12}$ Sein umfassender Abwägungshorizont kommt dem "ganzheitlichen " Prüfungsanspruch der UVP am ehesten entgegen. ${ }^{13}$ Der entscheidende Grund hierfür dürfte sein, daß es mit Konzentrationswirkung ausgestattet ist. Die Planfeststellung ersetzt alle behördlichen Entscheidungen (öffentlichrechtliche Genehmigungen, Verleihungen, Erlaubnisse, Bewilligungen, Zustimmungen und andere Planfeststellungen), die ansonsten erforderlich wären, damit das Vorhaben ausgeführt werden darf ( $\$ 75$ Abs. I VwVfG). Der Antragsteller braucht nicht bei einer Vielzahl von Behörden eine Vielzahl von Genehmigungen und sonstige Zulassungen einholen, um endlich die Anlage, die er errichten und betreiben will, genehmigt zu bekommen. Auch Drittbetroffene, die möglicherweise in ihrem rechtlich geschützten Interesse von dem Vorhaben tangiert werden, können umfassenden Rechtsschutz nicht erst in einer Vielzahl, sondern einem einzigen Verwaltungs- und anschließend in einem Gerichtsverfahren suchen.

Was man im Einzelnen unter der Konzentrationswirkung der Planfeststellung zu verstehen hat, ist umstritten. Klar scheint zunächst zu sein, daß die Kompetenzen der Behörden, deren Entscheidungen konzentriert werden, insoweit auf die Planfeststellungsbehörde übergehen, als diese deren eigentlich nötige Verwaltungsakte durch den Planfeststellungsbeschluß ersetzt. Unstreitig ist alsdann, daß das Verfahrensrecht der Planfeststellung anderweitige genehmigungsrechtliche Verfahrensnormen verdrängt. $\mathrm{Ob}$, wie und inwieweit indes die materiell-rechtlichen Vorschriften für die konzentrierten Verwaltungsakte die Planfeststellungsbehörde binden, ist noch nicht endgültig geklärt. ${ }^{14}$ Gleichwohl postuliert Breuer, vieles spreche für die sherrschende() und zutreffende() Ansicht «, daß »die materielle(?) Konzentrationswirkung der Planfeststellung die Bindung an das materielle Recht anderer Sachbereiche unberührt * lasse. ${ }^{15}$ Das bedeutet aber auch nach Breuer, daß die materielle Konzentrationswirkung das Planfeststellungsvorhaben nicht etwa privilegiert, sondern lediglich die verschiedenen Zulassungsakte in einem einheitlichen Gesamtakt zusammenfaßt. ${ }^{16}$ Ossenbühl weiß dagegen von einer anderen "gegenwärtig berrschenden Auffassung « zu berichten, nach der "sich die Theorie der formellen Konzentration durchgesetzt « hat. Die Planfeststellungsbehörde bleibt auch danach an materiell-rechtliche Regelungen gebunden, konzentriert bei sich aber die Zuständigkeit, das Verfahren sowie die Entscheidungsbefugnisse. ${ }^{17}$

11 Ausgenommen allerdings der 4. Abschnitt der AtVfV über die Genehmigung (mit Ausnahme der Zustellung durch offentliche Bekanntmachung n. \$17).

12 Vgl. dazu die Hinweise bei Gaentzsch, Die bergrechtliche Planfeststellung, FS Sendler (1991), S. 403 (405); Ronellenfitsch, Die Planfeststellung, VerwArch 80 (1989), 93 (97ff).

$13 \mathrm{Vgl}$. Gaentzsch (Fn. 12), S. $410 \mathrm{f}$.

14 Vgl. Steinberg, Das Nachbarrecht der öffentlichen Anlagen (1988), III. 189ff; Kühling, Fachplanungsrecht (1988), Rdn. $331 \mathrm{ff}$, jeweils m. w. N.

Is Breuer, Die Planfeststellung für Anlagen zur Endlagerung radioaktiver Abfälle (I984), S. 47

r6 Ebd.

17 Ossenbuhl, Verfassungsrechtliche Aspekte des atomrechtlichen Planfeststellungsverfahrens, FS Sendler (1991), S. 107 (109). 
Wegen ihrer Undeutlichkeiten haben die Aussagen zur Konzentrationswirkung im Konrad-Verfahren hohe politische Brisanz. Das BVerfG hat im Urteil zum niedersächsischen Weisungsstreit ausgeführt, daß die konkurrierende Gesetzgebungszuständigkeit nach Art. 74 Nr. I I a GG auch die Befugnis beinhaltet, die Umweltverträglichkeitsprüfung als unselbständigen Teil des Planfeststellungsverfahrens auszugestalten; die Prüfung und Bewertung der Umweltverträglichkeit unterliegen als Akte der Ausführung des AtG daher der Weisungskompetenz des Bundes und die gesamte Vollzugstätigkeit des Landes innerhalb der Auftragsverwaltung ist von der Weisungskompetenz des Bundes erfaßt. ${ }^{18}$

An diese Worte knüpft Ossenbübl an, wenn er - unermüdlich - argumentiert, aufgrund der Anordnung eines Planfeststellungsverfahrens durch das AtG werde der Umfang der Weisungsbefugnis des Bundes nach Art. 8s Abs. 3 GG in der atomrechtlichen Auftragsverwaltung durch den Umfang der Konzentrationswirkung des atomrechtlichen Planfeststellungsverfahrens umrissen. ${ }^{19}$

Dem BMU ist nach geltendem Atomrecht ohnehin eine recht komfortable Stellung zugedacht. Er kann das ihm nachgeordnete BfS anweisen, wie es Antrag und Genehmigungsunterlagen und -nachweise im Planfeststellungsverfahren zu formulieren habe und im Anschluß daran unter Bezug auf Art. 8s Abs. 3 GG die Planfeststellungsbehörde anweisen, wie sie darüber zu entscheiden habe. Kurz und knapp sagt das BVerfG hierzu: "Die Sachkompetenz, die er (gem.: der Bund, Anm. C.S-E) mit der Weisung in Anspruch nimmt, ist verfassungsrechtlich begründet. Sie wird nicht dadurch in Frage gestellt, daß der Bund durch den Antrag einer Bundesbebörde seinen gesetzlichen Pflichten zur atomaren Entsorgung nachkommt. «"

Nach Auffassung Ossenbübls gilt dies nun nicht allein für den Vollzug des AtG, sondern soll unbeschadet Art. 30 und 83 GG übertragbar sein auch auf den Vollzug jeglicher anderer Gesetzesmaterien, so sie nur planfeststellungsrechtlich konzentriert werden. Die Bundesaufsicht nach Art. 85 Abs. 4 GG erstreckt sich auf die Recht- und Zweckmäßigkeit der Gesetzesausführung durch das Land. Wird im atomrechtlichen Planfeststellungsverfahren z. B. das Wasserrecht konzentriert ${ }^{21}$, läuft Ossenbübls Auffassung, beim Wort genommen, darauf hinaus, der BMU könne von Bonn aus niedersächsische Gewässer bewirtschaften. ${ }^{22}$ Oder er könnte etwa über Varianten der Anbindung des Endlagers an die Straßen in Salzgitter befinden (vgl. etwa $\$ 20$ Abs. 7 NStrG ${ }^{23}$ ).

Soll die Bindung an das konzentrierte materielle Recht im Planfeststellungsverfahren mehr aussagen als die selbstverständliche Bindung der planfeststellenden Verwaltung an Recht und Gesetz, muß der dementsprechenden behördlichen Verwaltungsstruk-

I 8 BVerfGE 84, 25 (32)

I9 Ossenbühl, Novellierung des Atomgesetzes und Bundesauftragsverwaltung, in: Lukes (Hrg.), Reformüberlegungen zum Atomrecht (1991), S. 27 (73); ders., Verfassungsrechtliche Fragen - Bundesauftragsverwaltung, in: Lukes/Birkhofer (Hrg.), 9. AtRS (1991), S. 5I (65); ders. (Fn. 17), S. r 10 f.

20 BVerfGE 84, 25 (32).

2I Die Konzentration des Wasserrechts in Planfeststellungen wird aufgrund der besonderen Regelung des $\$ 14$ WHG kontrovers diskutiert, da diese Vorschrift auch als bewußte Herausnahme des Wasserrechts aus der Konzentrationswirkung interpretiert werden kann - so z. B. Gieseke/Wiedemann/Czychowski, WHG, $\$_{14}$ Anm. 2a; Breuer, Verfahrens- und Formfehler der Planfeststellung für raum- und umweltrelevante Großvorhaben, FS Sendler (1991), S. 357 (36r); a.A. Huntemann, Recht der unterirdischen Endlagerung radioaktiver Abfälle (1989), S. 95 f; Ossenbühl (Fn. I7), S. i i if.

22 Zwar verlangt $\$ 14$ Abs. 3 WHG für die wasserrechtlichen Entscheidungen das Einvernehmen der Wasserbehörde; ist die wasserrechtliche Entscheidung konzentriert, ist die explizite gesetzliche Anordnung des notwendigen Einvernehmens der Wasserbehorde mit entfallen und den Auslegungskünsten findiger Dogmatiker gegen den eindeutigen gesetzgeberischen Willen Tür und Tor geöffnet.

23 Niedersachsisches Straßengesetz (NStrG) i.d.F. vom 24. September 1980, Nds. GVBl. S. 359. 
tur Respekt gezollt werden. Nicht umsonst entscheiden Behörden zuständigkeitshalber lediglich über die Ausführung der Gesetze in ihrem Zuständigkeitsbereich, hat das doch auch etwas mit inhaltlicher, sachlicher Kompetenz zu tun. Und nicht zuletzt treffen für die planfeststellungsmäßig konzentrierten inhaltlichen Entscheidungen die von $O$ ssenbübl selbst rezipierten ${ }^{24}$ Gründe entweder nicht, oder allenfalls bedingt $\mathrm{zu}$, denen sich seinerzeit die Einführung der Bundesauftragsverwaltung bei Ausführung des AtG verdankt. ${ }^{25} \mathrm{Daß}$ die grundgesetzliche Beschränkung der gesetzlichen Kompetenz, Bundesauftragsverwaltung anzuordnen - immerhin war der Erlaß des AtG nicht nur mit der Änderung des Art. ${ }_{74}$ GG (Einführung der Nr. 1 Ia), sondern auch des Art. 87 GG (Einführung des Art. 87c) verbunden -, gleichfalls unterhöhlt wird, sei nur am Rande erwähnt. ${ }^{26}$

Insgesamt spricht vieles dafür, entweder lediglich von einer formellen Konzentration der Entscheidungen und insoweit der nur formellen Zuständigkeit für den Erlaß dieser Entscheidungen auszugehen ${ }^{27}$ und damit die Verantwortung für die materiellen Entscheidungsgehalte bei den im Planfeststellungsverfahren zu beteiligenden Behörden zu belassen. ${ }^{28}$ Oder man sollte zumindest die Integration der UVP in ein Genehmigungsverfahren und die Konzentrationswirkung der Planfeststellung hinsichtlich der Weisungskompetenz des Bundes in der Bundesauftragsverwaltung auseinanderhalten. ${ }^{29}$ Gleichwohl wird im Konfliktfall davon auszugehen sein, daß sich der BMU die Auffassung Ossenbübls zu eigen machen wird, hat er doch auch bisher keine Scheu gezeigt, vom Instrument der Weisung Gebrauch zu machen.

\section{Anlagenbegriff}

Aufgrund der nur begrenzten Konzentrationswirkung einer atomrechtichen Genehmigung (vgl. $\$ 8$ Abs. 2 AtG) mußte sich die Rechtsprechung schon mehrfach eingehender mit dem atomrechtlichen Anlagenbegriff auseinandersetzen. ${ }^{3} \mathrm{Da}$ zum Atomrecht parallele Genehmigungen, wie etwa bei der Errichtung eines Kernkraftwerkes, erforderlich sind ${ }^{31}$, entscheidet der atomrechtliche Anlagenbegriff ${ }^{32}$ über die Reichweite der atomrechtlichen Genehmigung. Dies gilt speziell für die Abgrenzung zu (anderen) baulichen Anlagen auf dem gleichen Gelände, ohne die zwar ein Kernkraftwerk nicht betrieben werden könnte, die gleichwohl für die kerntechnische

\footnotetext{
24 Vgl. Ossenbühl, 9. AtRS (Fn. 19), S. \$1 (52).

25 Ossenbühl führt hierzu an (ebd.):

- die Notwendigkeit einer zentralen Steuerung der überwachungsbedurftigen neuartigen Technik,

- die überregionale Bedeutung der Herstellung und Verwendung von Kernenergie,

- die Sicherstellung der internationalen Verpflichtungen der Bundesrepublik auf dem Gebiet der Kernenergie, sowie

- die Vernetzung mit anderen. Verwaltungsbereichen der Länder (z. B. im Wasserrecht, Baurecht, Naturschutz usw.)

Einzig der letzte Spiegelstrich konnte hier also einschlägig sein. Indes ist das mit Konzentration verbundene Planfeststellungsverfahren erst mit der 4 . Novelle zum AtG von 1976 eingeführt worden!

26 A.A. Ossenbihl (Fn. 17), S. $112 \mathrm{ff}$

27 Vgl. Stelkens/Bonk/Leonhardt, VwVfG, $\$ 75$ Anm. I I.

28 Dafür speziell im Hinblick auf die bergrechtliche Planfeststellung Bohne, Die Umweltvertraglichkeitsprüfung bergbaulicher Vorhaben, ZfB 130 (1989), 93 ( (1 I). Vgl. auch die explizite Einvernehmensregelung nach $\$ \mathrm{I}_{4} \mathrm{Abs} .3$ WHG.

29 Dafür (mit anderer Begründung) auch Steinberg, Verfassungsrechtliche Fragen einer Reform des Atomgesetzes, insbesondere der Verteilung der Verwaltungskompetenzen im Bundesstaat, 9. AtRS, S.67 (75 f).

30 S. insbes. die Urteile BVerwG, DVBI 1986, 190 (198) (Wyhl) und BVerwG, DVB1 1988, 973 (974) (Wackersdorf).

31 Vgl. zum einzelnen Kröncke, Die Genehmigung von Kernkraftwerken (1982).

32 Vgl. hierzu noch Bender/Sparwasser, Umweltrecht, Rdn. 5 I $_{3}$ ff; Klopfer, Umweltrecht $\$ 8$ Rdn. 22, jeweils $\mathrm{m}$. w. N
} 
Sicherheit im engeren Sinn nicht von Bedeutung sind. Dies ließ Ronellenfitsch zu der sprachlich etwas verunglückten Formulierung kommen: "Eine Anlage gemäß $\llbracket 7$ Abs. I At $G$ wird damit von ibren sicherheitstechnisch relevanten Anlagenteilen gebildet. «33

Die Anordnung der Planfeststellung für die Errichtung und den Betrieb eines Endlagers nötigt nicht zu einer ähnlichen Strapazierung des Anlagenbegriffs. Denn die Planfeststellung erhebt, wie erwähnt, einen umfassenden Regelungsanspruch für ein Vorhaben und soll es insgesamt in all seinen öffentlich-rechtlichen Facetten erfassen. Diese sind freilich auch allesamt und im Gegensatz zu parallelen Genehmigungsverfahren unterschiedslos der Öffentlichkeitsbeteiligung ausgesetzt. Von daher schadet es nicht, den Anlagenbegriff hier ähnlich weit wie im Abfallrecht zu fassen und lediglich auf die bestimmungsmäßige Nutzung der Anlage bzw. des zur Anlage dienenden Grundstücks abzuheben. ${ }^{34}$

Was jenseits dieses weit gefaßten Anlagenbegriffs liegt, muß dennoch nicht per se von der Planfeststellung unberührt bleiben. Nach $\$ 75$ Abs. 1 S. I VwVfG wird durch die Planfeststellung die Zulässigkeit des Vorhabens einschließlich der notwendigen Folgemaßnahmen an anderen Anlagen festgestellt. Wie unlängst das BVerwG hierzu entschieden hat, gehören dazu freilich nur solche Maßnahmen, die zum Anschluß und zur Anpassung des Vorhabens an andere Anlagen gehören; auch im Planfeststellungsverfahren sind die eigenen Planungskompetenzen und Zuständigkeiten anderer Behörden zu wahren. ${ }^{35}$ Planungskonkurrenzen regelt i.ü. $\$ 78 \mathrm{VwVfG}$.

\section{Atomrechtliches Endlager und BBergG}

Auffallend ist an dem vom BfS für die Öffentlichkeitsbeteiligung ausgelegten Plan, daß er das sog. Haufwerk, das bei der Auffahrung der Grubenbaue und Herrichtung der Einlagerungskammern für den atomaren Abfall im Schacht Konrad anfällt, praktisch nicht behandelt. Nur ein Satz im Abschnitt zum Bergwerksbetrieb (Ziffer 3.2.5 des Plans) informiert darüber, daß das zu Tage geförderte Gestein auf einer Teilfläche des Tagebaus Haverlahwiese deponiert werde, soweit es nicht anders verwertet werden kann. ${ }^{36}$

Diese Aufhaldung bedarf entsprechend dem Hinweis auf die mangelnde anderweitige Verwendungsmöglichkeit für das Material ihrerseits der abfallrechtlichen Planfeststellung oder -genehmigung nach $\mathbb{7} \mathrm{AbfG}$, wenn hier nicht die Herausnahme bergrechtlicher Abfälle aus dem AbfG gem. dessen $\$ 1$ Abs. 3 Nr. 3 greift.

\section{Das atomare Endlager als Bergwerk}

Die untertägige Verbringung radioaktiven Abfalls in eine geologische Formation erfordert die bergmännische Erschließung entsprechender Hohlräume, einen Bergwerksbetrieb, der dem BBergG mit seinem genehmigungsrechtlich spezifischen Instrumentarium des Betriebsplanverfahrens nach $₫$ soff BBergG unterworfen ist. Auch wenn bei unbefangener Betrachtung sich einiges dagegen sträubt, entspricht es doch der unangefochtenen Auffassung der Literatur, daß auch die reine Hohlraum-

33 Ronellenfitsch, Das atomrechtliche Genehmigungsverfahren (1983), S. 177

34 Vgl. Kunig/Schwermer/Versteyl, AbfG. $\$ 4$ Anm. I 2.

3s Vgl. BVerwG, DVBl 1988, 843; Kuhling, Fachplanungsrecht (1988), Rdn. 334 m.w. N.

36 BIS, Plan Endlager für radıoaktive Abfälle, Schachtanlage Konrad, Kurzfassung Stand 9/86, i. d. F. 4/90, S. 70 .

37 Vgl. G. Kühne, Rechtsfragen der Endlagerung radioaktiver Abfälle aus der Sicht des Bergrechts, ZfB I 32 (1991), 283 (284). Eingehend hierzu Huntemann (Fn. 2I), S. 1 23 ff 
schaffung zwecks späterer Einlagerung von Abfällen eine "Gewinnung von Bodenschätzen « i.S.d. $\$ 2$ Abs. I Nr. I i. V.m. $\$ 4$ Abs. 2 BBergG darstellt, sofern nur dabei in $\$ 3$ BBergG festgelegte Bodenschätze zutage gefördert werden. ${ }^{37}$ Denn das $\mathrm{BBergG}$ geht von einem objektiven Gewinnungsbegriff aus, der die spätere Verwertung und Verwendung der gewonnenen Bodenschätze dahingestellt sein läßt. ${ }^{38}$

Für den bergrechtlichen Laien scheint dann auf den ersten Blick $\$$ I26 Abs. 3 $\mathrm{BBergG}$ einschlägig zu sein. Er verlangt ein Betriebsplanverfahren nach $₫ 50 \mathrm{ff}$ BBergG für die Errichtung und den Betrieb einer Anlage zur Lagerung, Sicherstellung und Endlagerung radioaktiver Stoffe. Dagegen wird angeführt, sein Anwendungsbereich reduziere sich im wesentlichen auf die letzte Betriebsphase eines solchen Endlagers, wenn keine neuen Hohlräume mehr geschaffen werden. ${ }^{39}$ Ganz spitzfindig läßt sich zudem darauf verweisen, daß $\$ 126$ Abs. 3 BBergG außerdem noch die Eignung zur behälterlosen Speicherung zur Tatbestandsvoraussetzung erhebe, in das Endlager Konrad indes nur konditionierte, d. h. in Behältern verpackte Abfälle eingebracht werden sollen. ${ }^{\circ}$ Desungeachtet $z$ wingt auch die gleichzeitige Anwendbarkeit verschiedener Regelungen des BBergG nicht zu einer formalistischen Abgrenzung der jeweiligen Tatbestandsbereiche, sondern führt zur Anwendung des Gesetzes in "quasi eine $(r)$,Tateinheit «" ${ }^{41}$

Für die genehmigungsrechtliche Behandlung der Ablagerung des Haufwerks bedeutet dies, daß die Herausnahme aus dem Anwendungsbereich des AbfG nach $\mathbb{I}$ Abs. 3 Nr. 3 AbfG greift, wenn ein unmittelbarer betrieblicher Zusammenhang mit dem Bergwerksbetrieb besteht, dessen Bodenschätze, Nebengestein oder sonstigen Massen auf Halde genommen werden. Selbst die Beförderung über eine größere Entfernung zur Aufhaldung steht insoweit der Annahme des betrieblichen Zusammenhangs nicht entgegen..$^{2}$

Da $₫ 9$ b Abs. s Nr. 3 AtG das Bergrecht von der Konzentration der atomrechtlichen Planfeststellung ausnimmt, ist die Haufwerksdeponierung nicht in der atomrechtlichen Planfeststellung zu behandeln. Es gelten für sie allein die bergrechtlichen Bestimmungen. Daß sie deshalb auch für das Verfahren der Öffentlichkeitsbeteiligung ohne Belang ist, ist damit freilich nicht gesagt.

\section{Die bergrechtliche Umweltverträglichkeitsprüfung}

Die Umsetzung der EG-Richtlinie zur UVP hat mit dem Gesetz zur Änderung des BBergG vom I 2. Februar $1990^{43}$ auch Vorhaben nach dem BBergG der Prüfung und Bewertung ihrer Umweltverträglichkeit unterworfen. Dem dient insbesondere die eigens dafür neu in das Gesetz aufgenommene bergrechtliche Planfeststellung nach IS $57^{a-c}$ BBergG. 44

Welche bergrechtlichen Vorhaben im einzelnen UVP-pflichtig sind, regelt die Verordnung über die Umweltverträglichkeitsprüfung bergbaulicher Vorhaben (UVP-V Bergbau) vom I3. Juli 1990.45 Wegen der Doppelspurigkeit der Anwendung des Bergrechts auf die Anlage Schacht Konrad sind hier ebenfalls zwei Tatbestände ein-

$38 \mathrm{Vgl}$. Boldt/Weller, BBergG, $\$_{4}$ Anm. 7 .

39 G. Kühne (Fn. 37), S. 284.

40 S. Huntemann (Fn. 21 ), S. I I 9 ff.

$4^{1}$ Rengeling, Rechtsfragen zu Bundesendlagern für radioaktive Abfälje (1990), S. I9.

42 Vgl. Boldt/Weller, BBergG, $\$ 2$ Anm. 16.

43 BGBI I, S.215; nebst Materialien abgedruckt in ZfB 131 (1990), S.85ff. Eingehend hierzu Bohne (Fn. 26).

44 Vgl. Gaentzsch (Fn. I2); G. Kuhne, Die Einfuhrung der Umweltvertraglichkeitsprüfung im Bergrecht. UPR $1989,326 \mathrm{ff}$.

45 BGBI I, S. 1420; nebst Materialıen abgedruckt in ZfB 132 (1991), S. 99 ff. 

Stoffe).

Wenn auch das geltende Recht für den Schacht Konrad die UVP mehrfach (nach $\$ 9 b$ AtG und nach der UVP-V Bergbau) anordnet, findet aufgrund der Kollisionsregel des $\$ 57$ b Abs. 3 S. 2 BBergG nur eine UVP für diese Anlage statt. ${ }^{6}{ }^{6} \mathrm{Zwar}$ spricht hier der Gesetzestext allein von der bergrechtlichen UVP-Pflichtigkeit nach $\$$ I 26 Abs. 3 BBergG. Die Gesetzesbegründung gibt allerdings zu erkennen, daß sich der Gesetzgeber wohl nur $₫$ I 26 Abs. 3 BBergG als einschlägige bergrechtliche Norm für den Einbezug eines atomaren Endlagers in das Bergrecht vorstellen konnte. In Umkehrung der Grundregel, daß die bergrechtliche Planfeststellung mit UVP grundsätzlich Vorrang haben soll vor anderen parallelen Anordnungen von Planfeststellungsverfahren für die gleiche Anlage ( $\$ 57$ b Abs. 3 S. I BBergG), wollte er im atomrechtlichen Genehmigungsverfahren nach $\$ 9 \mathrm{~b}$ AtG dessen Vorrang gesichert sehen. ${ }^{47}$ Das wird auch gelten müssen, solange die UVP-Pflicht nicht auf $\$$ I Nr. 7 UVP-V Bergbau und damit auf der Anwendung des $\$$ I 26 Abs. 3 BBergG beruht, sondern auf $\$$ I Nr. I a UVP-V Bergbau unter Annahme eines Gewinnungsbetriebes nach $\$ 2$ Abs. I Nr. I i.V.m. $\$ 4$ Abs. 2 BBergG.

Offen ist noch, inwieweit diese Kollisionsregel den Ausschluß der Konzentration des Bergrechts nach $₫ 9$ b Abs. 5 Nr. 3 AtG relativiert oder diesem widerspricht. $4^{8}$ Das sei an dieser Stelle dahingestellt. Im Hinblick auf die Vorrangregelung ist hier nur festzuhalten, daß eine UVP in dem einen Verfahren nur dann durch die des anderen Verfahrens ersetzt werden kann, wenn sie sich wechselseitig decken. Die Vermeidung doppelter oder gar konkurrierender Verfahren und gleicher Prüfungen ist der Sinn der Kollisionsregeln des $\$ s>b$ Abs. 3 BBergG ${ }^{49}$, nicht jedoch die Reduktion des Prüfungsumfanges und -gegenstandes.

Soweit die Anlagenbegriffe in den jeweils parallel angeordneten Planfeststellungsverfahren nicht kongruent sind, muß für die UVP der Anlage insgesamt ein weiter Vorhabensbegriff ${ }^{\circ \circ}$ zugrundegelegt werden, der alle in das Vorhaben inkorporierte, sonst separate Verfahren umfaßt. Aus diesem Grund wird man auch beim Vorrang der UVP nach $₫ 9$ b AtG fordern müssen, daß das gesamte Endlagerbergwerk in die Prüfung mit einbezogen wird, einschließlich seiner Abraumhalde.

Von daher wird man wohl kaum umhin kommen festzustellen, daß die Unterlagen für die Öffentlichkeitsbeteiligung im Planfeststellungsverfahren Schacht Konrad in der Tat noch nicht auslegungsreif waren, als der BMU das NMU zur Einleitung des Verfahrens der Öffentlichkeitsbeteiligung anwies. Denn sie sparen einen ganzen, nicht unbedeutenden Teil des Vorhabens aus.

\section{Ausblick}

Bundesumweltminister Töpfer hegt große Erwartungen hinsichtlich des Erörterungstermins im Planfeststellungsverfahren Schacht Konrad. Gegenüber der Frankfurter Rundschau prophezeite er, dort "werde ein Beamter der Landesregierung, der das Projekt gern selbst verbindern möchte, die Anbörung vor einem abnehin kritischen Publikum abhalten. ... Eigentlich müsse er jemanden aus seinem Ministerium

$46 \mathrm{Boldt} / \mathrm{W}$ eller, BBergG, \$57c Anm. 10.

47 Vgl. die Wiedergabe der Gesetzesbegründung BT-Drs. 11/4015 in ZfB 131 (1990), 85 (98).

48 Vgl. Gaentzsch (Fn. I 2), S. 407 in Fn. 24 einerseits, Boldt/Weller, BBergG, $\$ s 7 c$ Anm. 10 andererseits.

49 Vgl. die Wiedergabe der Gesetzesbegründung BT-Drs. 11/4015 in ZfB 131 (1990), 85 (98).

so Zum Vorhabensbegriff der UVP vgl. Erbguth/Schink, UVPG, $\$ 2$ Anm. 4 s.

5I Frankfurter Rundschau v. 24. 2. 1992. 
zur Kontrolle daneben setzen, um zu verbindern, daß absichtlich Verfahrensfehler verursacht werden, was wiederum die Möglichkeit der Klage eröffnen würde "s."

Ganz abgesehen davon, ob die beamten- und disziplinarrechtlichen sowie schadensersatzrechtlichen Risiken eines solchen kollusiven Verhaltens für diesen »einen Beamten « tragbar wären - auch ansonsten scheint der Minister rechtlich nicht oder nur schlecht beraten gewesen zu sein, als er sich zu diesen Aussagen verstieg.

Bekanntlich kann in einem so "fehlersensiblen "Verfahren wie dem Planfeststellungsverfahren ${ }^{s 2}$ ein Verfahrensfehler nur in seltenen Fällen die Planfeststellung insgesamt gefährden. ${ }^{53}$ Das gründet in der Anknüpfung des verwaltungsgerichtlichen Rechtsschutzes Dritter an die Notwendigkeit zur Geltendmachung der Verletzung eigener subjektiver öffentlicher Rechte ( $\$ 4^{2}$ Abs. 2 VwGO), was für reine Verfahrensnormen nach der Rechtsprechung des BVerwG nur ausnahmsweise anzuerkennen ist. ${ }^{54}$

Der exzessive Gebrauch des Weisungsrechts durch den BMU hingegen mutet an, als wolle er selbst im Konrad-Verfahren die Grenzen des für die Rechtsprechung erträglichen austesten: Die Erörterung soll, erneut auf Weisung, stattfinden, obwohl die wissenschaftlichen Gutachten zu dem Projekt bis zum Erörterungstermin erst teilweise vorliegen. Was nicht weniger bedeutet, als daß sich erst dann die Behörde abschließend Klarheit über just diejenigen Umweltauswirkungen des Vorhabens verschafft haben kann, die sie schon vorher mit der verfahrensbeteiligten Öffentlichkeit erörtern soll. Auch wird vorab die zusammenfassende Darstellung und Bewertung des Vorhabens ( $\$ \rrbracket_{1} 1,12$ UVPG) vorzunehmen sein, um den Zeitrahmen des $\$ I 1 UVPG zu beachten. - Wie ernst werden hier Bürgerbeteiligung und UVP genommen, welchen Stellenwert haben sie für den BMU? Die Aussparung der Halde aus den Unterlagen, so ist zu vermuten, bildet für den BMU insoweit lediglich einen weiteren - freilich ins Bild passenden - faux pas.

\section{Jürgen Seifert \\ Vom Lauschangriff zum »Großen Lauschangriff« Darf es eine totale Überwachung der Wobnung geben?}

\section{Die Legalisierung des Lauschangriffs}

Am 4.6. 1992 hat der Deutsche Bundestag ein »Gesetz zur Bekämpfung des illegalen Rauschgifthandels und anderer Erscheinungsformen der Organisierten Kriminalität $(\mathrm{OrgKH})$ « verabschiedet. ${ }^{\mathrm{I}}$ In der vergangenen Legislaturperiode war ein solcher Gesetzentwurf (damals wie heute eingebracht vom Bundesrat) noch gescheitert. ${ }^{2}$ Mit dem verabschiedeten Gesetz werden Rechtsgrundlagen für den umstrittenen, aber seit Jahren praktizierten Einsatz von »Verdeckten Ermittlern« ( $\$$ I Ioa StPO)

52 Vgl. Breuer (Fn. 21), S. 357

s3 Es sei denn, dem verfahrensfehlerhaft zustandegekommenen Planfeststellungsbeschluß kommt enteignungsrechtliche Bedeutung zu, vgl. Kühling, Fachplanungsrecht (1988), Rdn. $399 \mathrm{ff}$.

54 Vgl. BVerwGE 61, 256(275); 64, 325; 75, 286(291); 85, 368 (373); BVerwG NJW 1992, 256 (257); Breuer (Fn. 2 r), S. $3^{87}$ f m. w. N.

1 Deutscher Bundestag, Stenobercht, 95. Sitzung, 4.6.1992, S. 7815; Drucksache 12/2720 u. 12/989. BGBl., I992, Teil I, S. $1302 \mathrm{ff}$.

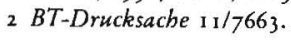

